\title{
Erratum to: An extreme learning machine model for the simulation of monthly mean streamflow water level in eastern Queensland
}

\author{
Ravinesh C. Deo • Mehmet Șahin
}

Published online: 7 March 2016

(C) Springer International Publishing Switzerland 2016

\section{Erratum to: Environ Monit Assess \\ DOI 10.1007/s10661-016-5094-9}

The accepted version of Table 6 for this published online article contained an error.

The corrected Table 6 is shown in the next page.

The online version of the original article can be found at http://dx. doi.org/10.1007/s10661-016-5094-9.

R. C. Deo $(\bowtie)$

School of Agricultural Computational and Environmental Sciences, International Centre of Applied Climate Science (ICACS), University of Southern Queensland, Springfield, QLD 4300, Australia

e-mail: ravinesh.deo@usq.edu.au

M. Șahin

Department of Electrical and Electronics Engineering, Siirt University, 56100 Siirt, Turkey 
Table 6 The model performance based on coefficient of determination $\left(R^{2}\right)$, Willmott's index $(d)$, Nash-Sutcliffe coefficient $\left(E_{\mathrm{NS}}\right)$, peak percentage deviation $\left(P_{\mathrm{dv}}\right)$ and mean absolute error (MAE) in the test period (2006-2012)

\begin{tabular}{|c|c|c|c|c|c|c|c|c|c|c|}
\hline \multirow[t]{2}{*}{ Station } & \multicolumn{5}{|l|}{ ELM } & \multicolumn{5}{|l|}{ ANN } \\
\hline & $R^{2}$ & $d$ & $E_{\mathrm{NS}}$ & $P_{\mathrm{dv}}(\%)$ & $\operatorname{MAE}(\mathrm{m})$ & $R^{2}$ & $d$ & $E_{\mathrm{NS}}$ & $P_{\mathrm{dv}}(\%)$ & $\operatorname{MAE}(\mathrm{m})$ \\
\hline \multicolumn{11}{|l|}{ Optimum Model } \\
\hline Gowrie Creek & 0.964 & 0.968 & 0.963 & 1.993 & 0.053 & 0.732 & 0.802 & 0.698 & 18.080 & 0.144 \\
\hline Albert River & 0.957 & 0.962 & 0.955 & -0.091 & 0.023 & 0.830 & 0.863 & 0.816 & -5.527 & 0.049 \\
\hline Mary River & 0.990 & 0.986 & 0.989 & 0.372 & 0.079 & 0.892 & 0.855 & 0.891 & -0.254 & 0.249 \\
\hline \multicolumn{11}{|c|}{ Trial Model with only rainfall and month as inputs } \\
\hline Gowrie Creek & 0.781 & 0.802 & 0.691 & 4.918 & 0.128 & 0.723 & 0.773 & 0.656 & 18.389 & 0.149 \\
\hline Albert River & 0.685 & 0.537 & 0.617 & -3.503 & 0.072 & 0.571 & 0.588 & -0.312 & 7.500 & 0.145 \\
\hline Mary River & 0.910 & 0.870 & 0.909 & 0.016 & 0.227 & 0.823 & 0.776 & 0.800 & 1.555 & 0.312 \\
\hline \multicolumn{11}{|c|}{ Trial Model all nine inputs (no prior selection) } \\
\hline Gowrie Creek & 0.732 & 0.809 & 0.698 & 18.080 & 0.144 & & & & & \\
\hline Albert River & 0.859 & 0.876 & 0.812 & 2.358 & 0.049 & & & & & \\
\hline Mary River & 0.928 & 0.903 & 0.926 & 4.178 & 0.222 & & & & & \\
\hline
\end{tabular}

The optimum model was selected based on input combinations $(x)$ and compared with an equivalent ANN model 